\title{
Adaptive Projective Synchronization between Two Different Fractional-Order Chaotic Systems with Fully Unknown Parameters
}

\author{
Liping Chen, ${ }^{1,2}$ Shanbi Wei, ${ }^{1}$ Yi Chai, ${ }^{1,2}$ and Ranchao $\mathrm{Wu}^{3}$ \\ ${ }^{1}$ School of Automation, Chongqing University, Chongqing 400044, China \\ ${ }^{2}$ State Key Laboratory of Power Transmission Equipment and System Security and New Technology, \\ Chongqing University, Chongqing 400044, China \\ ${ }^{3}$ School of Mathematics, Anhui University, Hefei 230039, China
}

Correspondence should be addressed to Shanbi Wei, weishanbi@126.com

Received 13 September 2011; Revised 20 November 2011; Accepted 20 November 2011

Academic Editor: Piermarco Cannarsa

Copyright (C) 2012 Liping Chen et al. This is an open access article distributed under the Creative Commons Attribution License, which permits unrestricted use, distribution, and reproduction in any medium, provided the original work is properly cited.

Projective synchronization between two different fractional-order chaotic systems with fully unknown parameters for drive and response systems is investigated. On the basis of the stability theory of fractional-order differential equations, a suitable and effective adaptive control law and a parameter update rule for unknown parameters are designed, such that projective synchronization between the fractional-order chaotic Chen system and the fractional-order chaotic Lü system with unknown parameters is achieved. Theoretical analysis and numerical simulations are presented to demonstrate the validity and feasibility of the proposed method.

\section{Introduction}

In the past three decades, the control and synchronization of chaotic systems have gained a lot of attention for their potential applications in some engineering application, such as image processing, chemical and biological systems and information science, and in particular in secure communication [1-5]. Since Pecora and Carroll introduced a method to realize complete synchronize between two identical chaotic with different initial conditions, several types of synchronization have been proposed, such as complete synchronization [6], lag synchronization [7], phase synchronization [8], antisynchronization [9], partial synchronization [10], generalization synchronization [11], impulsive synchronization [12], and so on. Among all kinds of chaos synchronization, projective synchronization phenomenon was first reported and discussed by González-Miranda [13]. In 1999, Mainieri 
and Rehacek first proposed the concept of projective synchronization in which drive and response systems could be synchronized up to a scaling factor [14]. It has been proven that projective synchronization is not in the category of generalized synchronization because the response system of projective synchronization is not asymptotically stable [15]. Recently, some scholars extended the concept of projective synchronization and proposed modified projective synchronization [16], function projective synchronization [17], and modified function projective synchronization [18]. Because of the proportionality between its synchronized dynamical states, the feature can be used to M-nary digital communication for achieving fast communication [19]. Projective synchronization has attracted increasing attention during recent years, and some necessary conditions for projective synchronization have been obtained, see references [20-23].

In the investigation of dynamical systems, many physical models could be properly described by the fractional order systems, see, for example, dielectric polarization, electrodeelectrolyte polarization, electromagnetic waves, viscoelastic systems, quantitative finance and diffusion wave [24-26]. Compared with the classical integer-order models, fractional order derivatives provide an excellent instrument for the description of memory and hereditary properties of various materials and processes. With the introduction of fractional derivatives, it was proved that many fractional-order differential systems behave chaotically with order less than 3, such as fractional-order Chua's circuit system [27], fractionalorder Rössler system [28], fractional-order Chen system [29], fractional-order Lü system [30] and fractional-order modified Duffing system [31]. In recent years, the control and synchronization of fractional-order chaotic systems have caught much attention, see references [32-34]. However, there exists a substantial difference between fractional-order differential systems and integer-order differential ones. Most of the properties, conclusions, and methods for dealing with the integer-order systems cannot be simply extended to the case of the fractional-order ones, such as Lyapunovs direct method. Therefore, results bout the synchronization of fractional-order chaotic systems are much less rich than those of integerorder systems. It is still a challenging problem.

On the other hand, at present, most of the theoretical results concerning synchronization of the fractional-order systems mainly focus on systems whose models are identical or similar, and parameters are exactly known in advance. But in many practical situation, the parameters of many systems cannot be known entirely, the synchronization will be greatly affected by these uncertainties. How to effectively synchronize two fractional-order chaotic systems with unknown parameters is an important problem for the theoretical and practical applications. As we know, we usually take adaptive control methods to deal with such problems. However, to our best knowledge, much of the literature on projective synchronization of fractional-order chaotic systems all focused on the case of parameter determination, for instances [35-38]. There are few results about synchronization of different fractional-order chaotic systems with fully unknown parameters [39], not to mention projective synchronization. Motivated by the above discussions, in this paper, we study projective synchronization between fractional-order chaotic Chen system and fractionalorder chaotic Lü system with fully unknown parameters in the derive system and the response one, which is more general and practical than the one discussed in paper [39], where only master systems are assumed to be uncertainty and a novel parameter identification and an adaptive control law are derived based on the stability theory of fractional-order differential equations. Corresponding theoretical analysis and numerical simulations are presented to verify the validity and feasibility of the proposed method. 
The remainder of this paper is organized as follows. In Section 2, preliminary results are presented and fractional-order chaotic Chen system and fractional-order chaotic Lü system are described. In Section 3, adaptive synchronization scheme with a parameter update law is presented. In Section 4, numerical simulations are given to illustrate the effectiveness of the main results. Finally, conclusions are drawn in Section 5.

\section{Preliminaries and System Description}

The fractional-order integrodifferential operator is the extending concept of integer-order integrodifferential operator, which can be denoted by a general fundamental operator as follows:

$$
{ }_{a} D_{t}^{q}= \begin{cases}\frac{d^{q}}{d t^{q}}, & R(q)>0 \\ 1, & R(q)=0 \\ \int_{a}^{t}(d \tau)^{-q}, & R(q)<0\end{cases}
$$

where $q$ is the fractional-order, which can be a complex number and $a$ and $t$ are the limits of the operation. There are some definitions for fractional derivatives. The commonly used definitions are Grunwald-Letnikov (GL), Riemann-Liouville (RL), and Caputo (C) definitions.

The Grunwald-Letnikov (GL) derivative with fractional-order $q$ is given by

$$
{ }_{a}^{\mathrm{GL}} D_{t}^{q} f(t)=\lim _{h \rightarrow 0} f_{h}^{(q)}(t)=\lim _{h \rightarrow 0} h^{-q} \sum_{i=0}^{[t-q / h]}(-1)^{i}\left(\begin{array}{l}
q \\
i
\end{array}\right) f(t-i h),
$$

where $[\cdot]$ means the integer part.

The Riemann-Liouvill (RL) fractional derivatives are defined by

$$
a^{R} D_{t}^{q} f(t)=\frac{d^{n}}{d t^{n}} \frac{1}{\Gamma(n-q)} \int_{a}^{t} \frac{f(\tau)}{(t-\tau)^{(q-n+1)}} d \tau, \quad n-1<q<n
$$

where $\Gamma(\cdot)$ is the gamma function and $\Gamma(\tau)=\int_{0}^{\infty} t^{\tau-1} e^{-t} d t$.

The Caputo (C) fractional derivative is defined as follows:

$$
\frac{1}{\Gamma(n-q)} \int_{a}^{t}(t-\tau)^{(n-q-1)} d \tau \longrightarrow \frac{1}{\Gamma(n-q)} \int_{a}^{t}(t-\tau)^{(n-q-1)} f^{(n)}(\tau) d \tau
$$

It should be noted that the advantage of Caputo approach is that the initial conditions for fractional differential equations with Caputo derivatives take on the same form as for integer-order differential, which have well understood physical meanings. Comparing these two formulas, one easily arrives at a fact that Caputo derivative of a constant is equal to zero, which is not the case for the Riemann-Liouville derivative. Therefore, in the rest of this paper, 
the notation $D_{*}^{q}$ is chosen as the Caputo fractional derivative operator ${ }_{a}^{C} D$. For more about knowledge of fractional definitions and fractional calculus, see references [40,41].

In the following section, an improved predictor-corrector algorithm [42] for fractionalorder differential equations is presented in brief, which will be used in numerical simulation section. In comparison with the classical one-step Adams-Bashforth-Moulton algorithm, the numerical approximation of the improved algorithm is more accurate and the computational cost is more lower. Predictor-corrector algorithm for fractional differential equations is the generalization of Adams-Bashforth-Moultono one; the similarities, differences, merits, and demerits between them are shown in $[42,43]$.

Consider the following differential equation:

$$
\begin{gathered}
D_{*}^{q} x(t)=f(t, x), \quad 0 \leq t \leq T, \\
x^{(k)}(0)=x_{0}^{(k)}, \quad k=0,1,2, \ldots,[q]-1,
\end{gathered}
$$

equivalent to the Volterra integral equation [44]

$$
x(t)=\sum_{k=0}^{[q]-1} x_{0}^{(k)} \frac{t^{k}}{k !}+\frac{1}{\Gamma(q)} \int_{0}^{t} \frac{f(\tau, x)}{(t-\tau)^{1-q}} d \tau .
$$

Set $h=T / N, t_{n}=n h(n=0,1,2, \ldots, N)$. Then (2.6) can be discretized as follows:

$$
x_{h}\left(t_{n+1}\right)=\sum_{k=0}^{[q]-1} x_{0}^{(k)} \frac{t_{n+1}^{k}}{k !}+\frac{h^{q}}{\Gamma(q+2)} f\left(t_{n+1}, x_{h}^{\rho}\left(t_{n+1}\right)\right)+\frac{h^{q}}{\Gamma(q+2)} \sum_{j=0}^{n} a_{j, n+1} f\left(t_{j}, x_{h}\left(t_{j}\right)\right),
$$

where

$$
\begin{gathered}
a_{j, n+1}= \begin{cases}n^{q+1}-(n-q)(n+1)^{q}, & j=0, \\
(n-j+2)^{q+1}+(n-j)^{q+1}-2(n-j+1)^{q+1}, & 1 \leq j \leq n, \\
1, & j=n+1,\end{cases} \\
x_{h}^{\rho}\left(t_{n+1}\right)=\sum_{k=0}^{[q]-1} x_{0}^{(k)} \frac{t_{n+1}^{k}}{k !}+\frac{1}{\Gamma(q)} \sum_{j=0}^{n} b_{j, n+1} f\left(t_{j}, x_{h}\left(t_{j}\right)\right),
\end{gathered}
$$

in which $b_{j, n+1}=\left(h^{q} / q\right)\left((n-j+1)^{q}-(n-j)^{q}\right)$.

The error estimate $e$ is $\operatorname{Max}\left|x\left(t_{j}\right)-x_{h}\left(t_{j}\right)\right|=O\left(h^{\rho}\right)(j=0,1, \ldots, N)$, where $\rho=$ $\operatorname{Min}(2,1+q)$.

In 2004, Li and Peng [29] and Li and Chen [45] studied the chaotic behaviors of chaotic Chen system with fractional-order, which is described as follows:

$$
\begin{aligned}
& D_{*}^{q} x_{1}(t)=a\left(x_{2}-x_{1}\right), \\
& D_{*}^{q} x_{2}(t)=(c-a) x_{1}-x_{1} x_{3}+c x_{2}, \\
& D_{*}^{q} x_{3}(t)=x_{1} x_{2}-b x_{3},
\end{aligned}
$$

where $q$ is the fractional order, $0<q<1, x_{1}, x_{2}$ and $x_{3}$ are state variables, $a, b$ and $c$ are unknown parameters to be identified. When $a=35, b=3, c=28$ and $q=0.9$, system (2.9) 


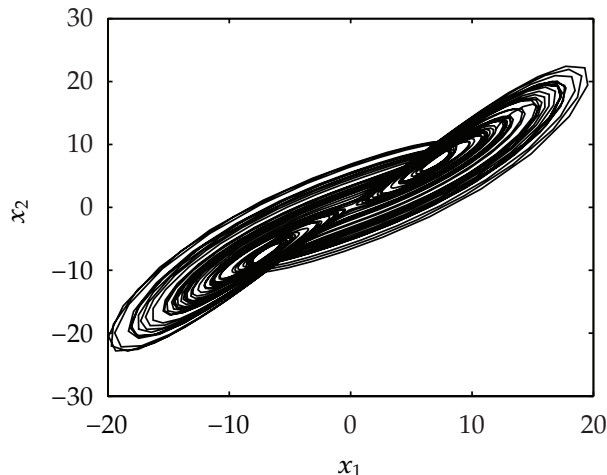

(a)

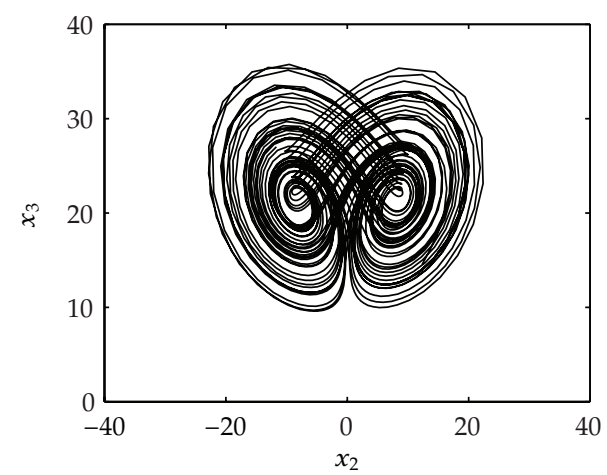

(c)

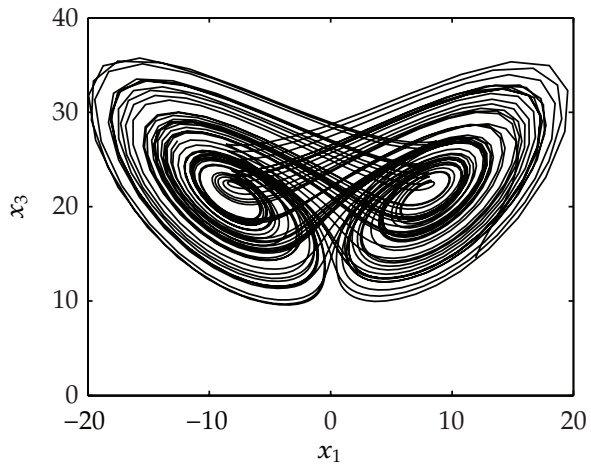

(b)

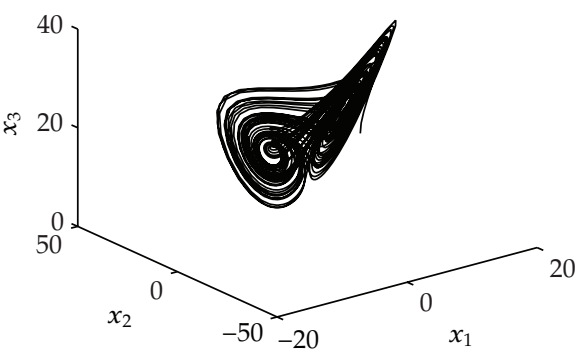

(d)

Figure 1: Chaotic attractors of fractional-order chaotic Chen system with order $q=0.9$.

exhibits chaotic behaviors as shown in Figure 1. References [46, 47] considered the synchronization of fractional-order chaotic Chen systems with determined parameters.

Meanwhile, Deng and Li [30] and Lu [48] numerically investigated the chaotic behaviors of the fractional-order Lü system, respectively. Fractional-order chaotic Lü system is given by

$$
\begin{aligned}
& D_{*}^{q} y_{1}(t)=\alpha\left(y_{2}-y_{1}\right), \\
& D_{*}^{q} y_{2}(t)=-y_{1} y_{3}+\beta y_{2}, \\
& D_{*}^{q} y_{3}(t)=y_{1} y_{2}-\theta y_{3},
\end{aligned}
$$

where $q$ is the fractional order; $0<q<1 ; y_{1}, y_{2}$, and $y_{3}$ are state variables; $\alpha, \beta$, and $\theta$ are unknown parameters to be estimated. System (2.10) exhibits a chaotic attractor for $\alpha=$ $36, \beta=20$, and $\theta=3$. Figure 2 displays the chaotic attractor of the fractional-order Lü chaotic system with the order $q=0.9$.

To obtain our results, the following Lemma is presented. For a given autonomous linear system of fractional order

$$
D_{*}^{q} x=A x,
$$

with $x(0)=x_{0}$, where $x \in R^{n}$ is the state vector, one has the following Lemma. 


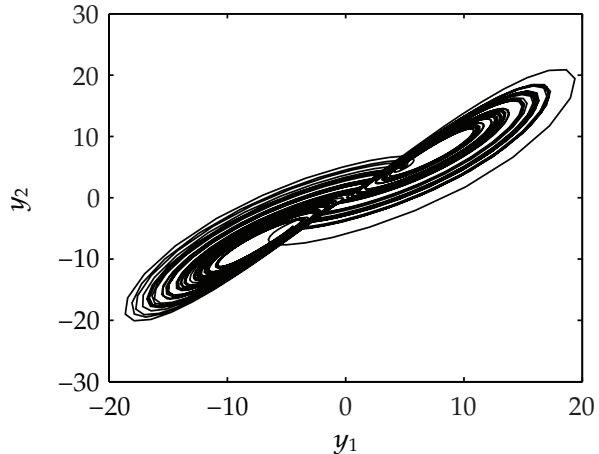

(a)

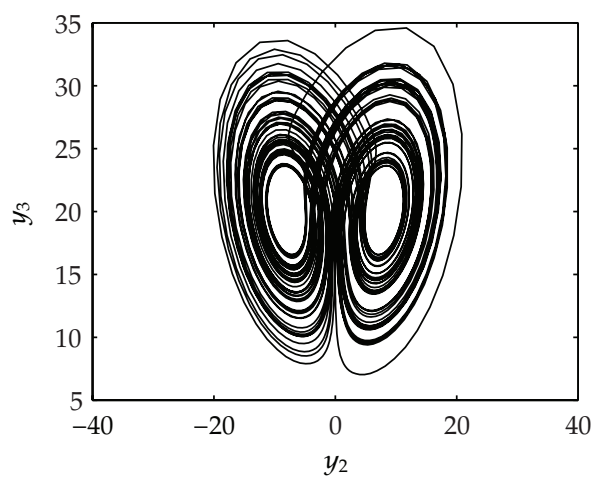

(c)

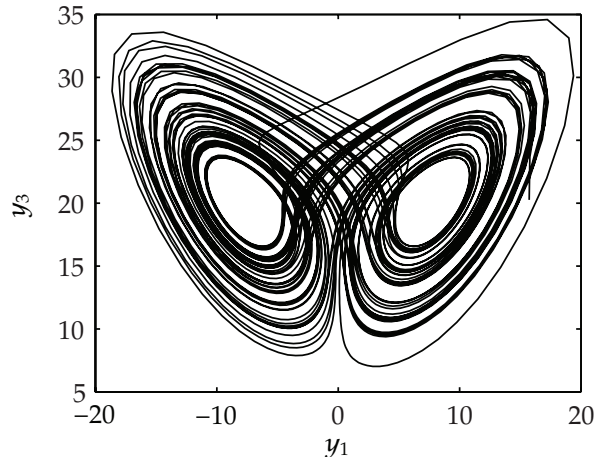

(b)

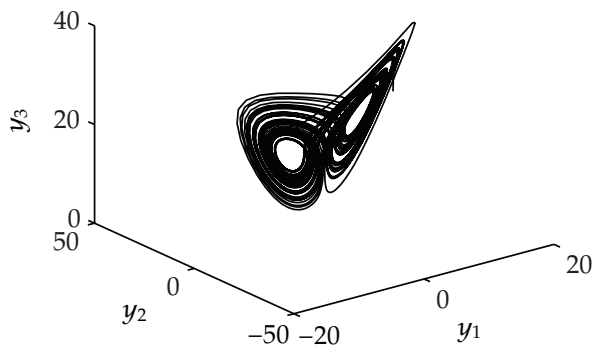

(d)

Figure 2: Chaotic attractors of fractional-order chaotic Lü system with order $q=0.9$.

Lemma 2.1 (see [49]). System (2.11) is

(i) asymptotically stable if and only if $\left|\arg \left(\lambda_{i}(A)\right)\right|>\alpha \pi / 2, i=1,2,3, \ldots$, where $\arg \left(\lambda_{i}(A)\right)$ denotes the argument of the eigenvalue $\lambda_{i}$ of $A$. In this case, the component of the state decays towards 0 like $t^{-\alpha}$;

(ii) stable if and only if either it is asymptotically stable or those critical eigenvalues that satisfy $\left|\arg \left(\lambda_{i}(A)\right)\right|=\alpha \pi / 2$ have geometric multiplicity one. Figure 3 illustrates the stability region of the fractional-order system.

\section{Adaptive Projective Synchronization}

In the section, we will discuss adaptive projective synchronization behavior between fractional-order chaotic Chen system and fractional-order chaotic Lü system with fully unknown parameters. Assume that fractional-order chaotic Chen system with three 


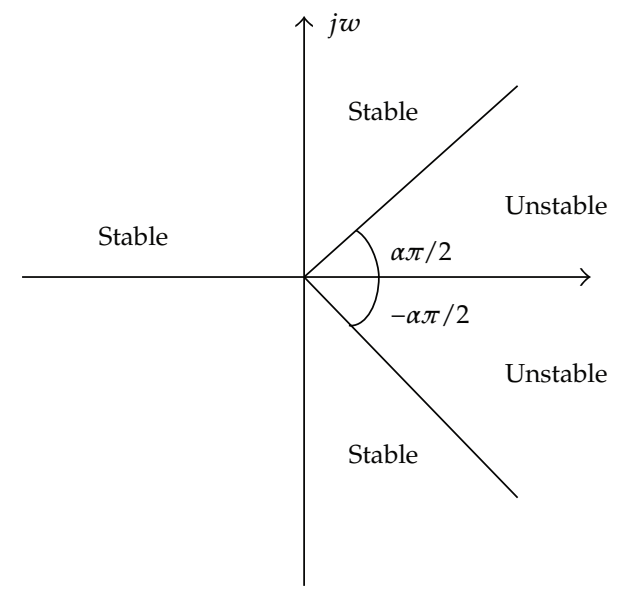

Figure 3: Stability region of the fractional-order system.

unknown parameters is the drive system and the response system is fractional-order chaotic Lü system, which are given as follows, respectively,

$$
\begin{aligned}
& D_{*}^{q} x_{1}(t)=a\left(x_{2}-x_{1}\right), \\
& D_{*}^{q} x_{2}(t)=(c-a) x_{1}-x_{1} x_{3}+c x_{2}, \\
& D_{*}^{q} x_{3}(t)=x_{1} x_{2}-b x_{3}, \\
& D_{*}^{q} y_{1}(t)=\alpha\left(y_{2}-y_{1}\right)+u_{1}, \\
& D_{*}^{q} y_{2}(t)=-y_{1} y_{3}+\beta y_{2}+u_{2}, \\
& D_{*}^{q} y_{3}(t)=y_{1} y_{2}-\theta y_{3}+u_{3},
\end{aligned}
$$

where $x_{i}$ and $y_{i}(i=1,2,3)$ stand for state variables of the master system and the slave one, respectively; $a, b, c, \alpha, \beta$, and $\theta$ are unknown parameters, which need to be estimated; $u_{1}, u_{2}$, and $u_{3}$ are nonlinear controllers to be designed later.

Definition 3.1. Systems (3.1) and (3.2) are adaptive projective synchronization if there exists a scaling matrix $\sigma$ such that

$$
\lim _{t \rightarrow \infty}\|e(t)\|=\lim _{t \rightarrow \infty}\|y-\sigma x\|=0
$$

where $\|\cdot\|$ is the Euclidean norm and $\sigma=\operatorname{diag}\left(\sigma_{1}, \sigma_{2}, \sigma_{3}\right)$.

It follows from (3.1)-(3.3) that we have the following error dynamical system:

$$
\begin{aligned}
& D_{*}^{q} e_{1}(t)=\alpha\left(y_{2}-y_{1}\right)-\sigma_{1} a\left(x_{2}-x_{1}\right)+u_{1}, \\
& D_{*}^{q} e_{2}(t)=-y_{1} y_{3}+\beta y_{2}-\sigma_{2}(c-a) x_{1}+\sigma_{2} x_{1} x_{3}-\sigma_{2} c x_{2}+u_{2}, \\
& D_{*}^{q} e_{3}(t)=y_{1} y_{2}-\theta y_{3}-\sigma_{3} x_{1} x_{2}+\sigma_{3} b x_{3}+u_{3},
\end{aligned}
$$

where $e_{1}=y_{1}-\sigma_{1} x_{1}, e_{2}=y_{2}-\sigma_{2} x_{2}$, and $e_{3}=y_{3}-\sigma_{3} x_{3}$. Our aim is to find suitable control laws $u_{i}(i=1,2,3)$ with a parameter estimation update law such that the response system (3.2) synchronizes the drive system (3.1) up to a scaling factor $\sigma$. 
To this end, the following criterion is proposed to ensure that the response system (3.2) effectively synchronizes the drive system (3.1) up to a scaling factor $\sigma$.

Theorem 3.2. For a given constant scaling matrix $\sigma=\operatorname{diag}\left(\sigma_{1}, \sigma_{2}, \sigma_{3}\right)$ and any initial conditions, projective synchronization between systems (3.1) and (3.2) will occur by the following adaptive controllers:

$$
\begin{aligned}
& u_{1}=(\tilde{\alpha}-1) e_{1}+\left(y_{3}-\tilde{\alpha}\right) e_{2}-y_{2} e_{3}+\tilde{\alpha}\left(\sigma_{1} x_{1}-\sigma_{2} x_{2}\right)+\tilde{a} \sigma_{1}\left(x_{2}-x_{1}\right) \\
& u_{2}=-(\tilde{\beta}+1) e_{2}+\left(\sigma_{1} \sigma_{3}-\sigma_{2}\right) x_{1} x_{3}-\sigma_{2} x_{2}(\tilde{\beta}-\tilde{c})+\sigma_{2} x_{1}(\tilde{c}-\tilde{a}) \\
& u_{3}=(\tilde{\theta}-1) e_{3}+\left(\sigma_{3}-\sigma_{1} \sigma_{2}\right) x_{1} x_{2}+\sigma_{3} x_{3}(\tilde{\theta}-\tilde{b})
\end{aligned}
$$

and the parameter update rules for six unknown parameters $a, b, c, \alpha, \beta$, and $\theta$

$$
\begin{aligned}
& D_{*}^{q} \tilde{a}=\left(\sigma_{1} x_{1}-\sigma_{1} x_{2}\right) e_{1}+\sigma_{2} x_{1} e_{2}, \\
& D_{*}^{q} \tilde{b}=\sigma_{3} x_{3} e_{3} \\
& D_{*}^{q} \tilde{c}=-\left(\sigma_{2} x_{1}+\sigma_{2} x_{2}\right) e_{2} \\
& D_{*}^{q} \tilde{\alpha}=\left(y_{2}-y_{1}\right) e_{1} \\
& D_{*}^{q} \tilde{\beta}=y_{2} e_{2} \\
& D_{*}^{q} \tilde{\theta}=-y_{3} e_{3}
\end{aligned}
$$

where $e_{a}=\tilde{a}-a, e_{b}=\tilde{b}-b, e_{c}=\tilde{c}-c, e_{\alpha}=\tilde{\alpha}-\alpha, e_{\beta}=\tilde{\beta}-\beta, e_{\theta}=\tilde{\theta}-\theta, \tilde{a}, \tilde{b}, \tilde{c}, \tilde{\alpha}, \tilde{\beta}$, and $\tilde{\theta}$ are the parameter estimations of $a, b, c, \alpha, \beta$, and $\theta$, respectively, and $e_{a}, e_{b}, e_{c}, e_{\alpha}, e_{\beta}$, and $e_{\theta}$ are the corresponding parameter errors.

Proof. Substituting (3.5) into (3.4) leads to the following error system:

$$
\begin{aligned}
& D_{*}^{q} e_{1}(t)=-e_{1}+y_{3} e_{2}-y_{2} e_{3}+\left(\sigma_{1} x_{2}-\sigma_{1} x_{1}\right) e_{a}+\left(y_{1}-y_{2}\right) e_{\alpha}, \\
& D_{*}^{q} e_{2}(t)=-y_{3} e_{1}-e_{2}-\sigma_{1} x_{1} e_{3}-\sigma_{2} x_{1} e_{a}+\left(\sigma_{2} x_{1}+\sigma_{2} x_{2}\right) e_{c}-y_{2} e_{\beta}, \\
& D_{*}^{q} e_{1}(t)=y_{2} e_{1}+\sigma_{1} x_{1} e_{2}-e_{3}-\sigma_{3} x_{3} e_{b}+y_{3} e_{\theta} .
\end{aligned}
$$

Combining (3.6) with (3.7), one has

$$
\begin{aligned}
& {\left[D_{*}^{q} e_{1}, D_{*}^{q} e_{2}, D_{*}^{q} e_{3}, D_{*}^{q} e_{a}, D_{*}^{q} e_{b}, D_{*}^{q} e_{c}, D_{*}^{q} e_{\alpha}, D_{*}^{q} e_{\beta}, D_{*}^{q} e_{\theta}\right]^{T}} \\
& \quad=A\left[e_{1}, e_{2}, e_{3}, e_{a}, e_{b}, e_{c}, e_{\alpha}, e_{\beta}, e_{\theta}\right]^{T},
\end{aligned}
$$


where

$$
A=\left[\begin{array}{ccccccccc}
-1 & y_{3} & -y_{2} & M & 0 & 0 & y_{1}-y_{2} & 0 & 0 \\
-y_{3} & -1 & -\sigma_{1} x_{1} & -\sigma_{2} x_{1} & 0 & N & 0 & -y_{2} & 0 \\
y_{2} & \sigma_{1} x_{1} & -1 & 0 & -\sigma_{3} x_{3} & 0 & 0 & 0 & y_{3} \\
-M & \sigma_{2} x_{1} & 0 & 0 & 0 & 0 & 0 & 0 & 0 \\
0 & 0 & \sigma_{3} x_{3} & 0 & 0 & 0 & 0 & 0 & 0 \\
0 & -N & 0 & 0 & 0 & 0 & 0 & 0 & 0 \\
y_{2}-y_{1} & 0 & 0 & 0 & 0 & 0 & 0 & 0 & 0 \\
0 & y_{2} & 0 & 0 & 0 & 0 & 0 & 0 & 0 \\
0 & 0 & -y_{3} & 0 & 0 & 0 & 0 & 0 & 0
\end{array}\right],
$$

Suppose $\lambda$ is one of the eigenvalues of matrix $A$ and the corresponding noneigenvector is $\varepsilon=\left(\varepsilon_{1}, \varepsilon_{2}, \varepsilon_{3}, \varepsilon_{4}, \varepsilon_{5}, \varepsilon_{6}, \varepsilon_{7}, \varepsilon_{8}, \varepsilon_{9}\right)^{T}$, that is,

$$
A \varepsilon=\lambda \varepsilon
$$

Take conjugate transpose $(H)$ on both sides of (3.10); one obtains

$$
\overline{(A \varepsilon)^{T}}=\bar{\lambda} \varepsilon^{H} .
$$

Using (3.10) multiplied left by $(1 / 2) \varepsilon^{H}$ plus (3.11) multiplied right by $(1 / 2) \varepsilon$, we derive

$$
\varepsilon^{H}\left(\frac{1}{2} A+\frac{1}{2} A^{H}\right) \varepsilon=\frac{1}{2}(\lambda+\bar{\lambda}) \varepsilon^{H} \varepsilon
$$

From (3.12), we have

$$
\frac{1}{2}(\lambda+\bar{\lambda})=\frac{\varepsilon^{H}\left((1 / 2) A+(1 / 2) A^{H}\right) \varepsilon}{\varepsilon^{H} \varepsilon} .
$$

By substituting $A$ into the right of (3.13), we can obtain

$$
\frac{1}{2}(\lambda+\bar{\lambda})=\frac{1}{\varepsilon^{H} \mathcal{\varepsilon}} \varepsilon^{H}\left[\begin{array}{ccccccccc}
-1 & 0 & 0 & 0 & 0 & 0 & 0 & 0 & 0 \\
0 & -1 & 0 & 0 & 0 & 0 & 0 & 0 & 0 \\
0 & 0 & -1 & 0 & 0 & 0 & 0 & 0 & 0 \\
0 & 0 & 0 & 0 & 0 & 0 & 0 & 0 & 0 \\
0 & 0 & 0 & 0 & 0 & 0 & 0 & 0 & 0 \\
0 & 0 & 0 & 0 & 0 & 0 & 0 & 0 & 0 \\
0 & 0 & 0 & 0 & 0 & 0 & 0 & 0 & 0 \\
0 & 0 & 0 & 0 & 0 & 0 & 0 & 0 & 0 \\
0 & 0 & 0 & 0 & 0 & 0 & 0 & 0 & 0
\end{array}\right] \varepsilon
$$


From (3.14), we have $\lambda+\bar{\lambda} \leq 0$, that is, any eigenvalue of matrix $A$ satisfies

$$
|\arg (\lambda)| \geq \frac{\pi}{2}>\frac{\alpha \pi}{2}(\alpha<1)
$$

Based on the stability theory of fractional-order systems (Lemma 2.1), the equilibrium point in error system (3.8) is asymptotically stable, that is,

$$
\lim _{t \rightarrow \infty}\|e(t)\|=\lim _{t \rightarrow \infty}\|y-\sigma x\|=0
$$

which implies that the adaptive projective synchronization between systems (3.1) and (3.2) can be achieved.

If we choose the scaling matrix $\sigma$ as $I$ and $-I$ ( $I$ is an $n \times n$ identity matrix), then we have the following Corollaries 3.3 and 3.4 to guarantee system (3.1) synchronizes system (3.2) in complete and inverse manner, respectively.

Corollary 3.3. If the adaptive controllers are chosen as

$$
\begin{aligned}
& u_{1}=(\tilde{\alpha}-1) e_{1}+\left(y_{3}-\tilde{\alpha}\right) e_{2}-y_{2} e_{3}+\left(x_{1}-x_{2}\right)(\tilde{\alpha}-\tilde{a}), \\
& u_{2}=-(\tilde{\beta}+1) e_{2}-x_{2}(\tilde{\beta}-\tilde{c})+x_{1}(\tilde{c}-\tilde{a}), \\
& u_{3}=(\tilde{\theta}-1) e_{3}+x_{3}(\tilde{\theta}-\tilde{b}),
\end{aligned}
$$

and update laws of parameters are chosen as

$$
\begin{aligned}
& D_{*}^{q} \tilde{a}=\left(x_{1}-x_{2}\right) e_{1}+x_{1} e_{2}, \\
& D_{*}^{q} \tilde{b}=x_{3} e_{3}, \\
& D_{*}^{q} \tilde{c}=-\left(x_{1}+x_{2}\right) e_{2}, \\
& D_{*}^{q} \tilde{\alpha}=\left(y_{2}-y_{1}\right) e_{1}, \\
& D_{*}^{q} \tilde{\beta}=y_{2} e_{2}, \\
& D_{*}^{q} \tilde{\theta}=-y_{3} e_{3},
\end{aligned}
$$

then complete synchronization between systems (3.1) and (3.2) will be achieved for any initial values, where $e_{a}=\tilde{a}-a, e_{b}=\tilde{b}-b, e_{c}=\tilde{c}-c, e_{\alpha}=\tilde{\alpha}-\alpha, e_{\beta}=\tilde{\beta}-\beta, e_{\theta}=\tilde{\theta}-\theta, \tilde{a}, \tilde{b}, \tilde{c}, \tilde{\alpha}, \tilde{\beta}$, and $\tilde{\theta}$ are the parameter estimations of $a, b, c, \alpha, \beta$, and $\theta$, respectively, and $e_{a}, e_{b}, e_{c}, e_{\alpha}, e_{\beta}$, and $e_{\theta}$ are the corresponding parameter errors.

Corollary 3.4. If the adaptive controllers are chosen as

$$
\begin{aligned}
& u_{1}=(\tilde{\alpha}-1) e_{1}+\left(y_{3}-\tilde{\alpha}\right) e_{2}-y_{2} e_{3}-\left(x_{1}-x_{2}\right)(\tilde{\alpha}-\tilde{a}), \\
& u_{2}=-(\tilde{\beta}+1) e_{2}+2 x_{1} x_{3}+x_{2}(\tilde{\beta}-\tilde{c})-x_{1}(\tilde{c}-\tilde{a}), \\
& u_{3}=(\tilde{\theta}-1) e_{3}-2 x_{1} x_{2}-x_{3}(\tilde{\theta}-\tilde{b})
\end{aligned}
$$




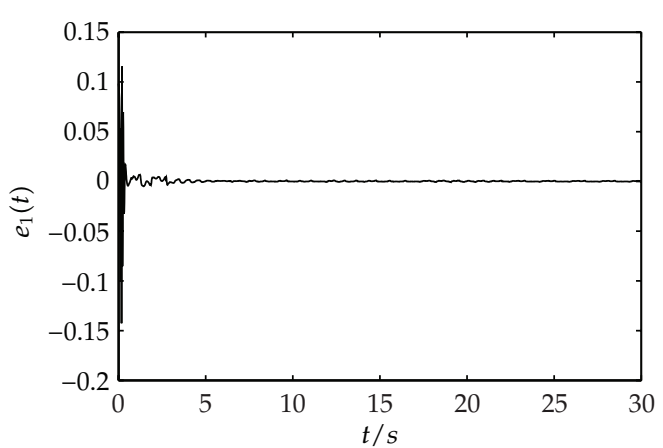

(a)

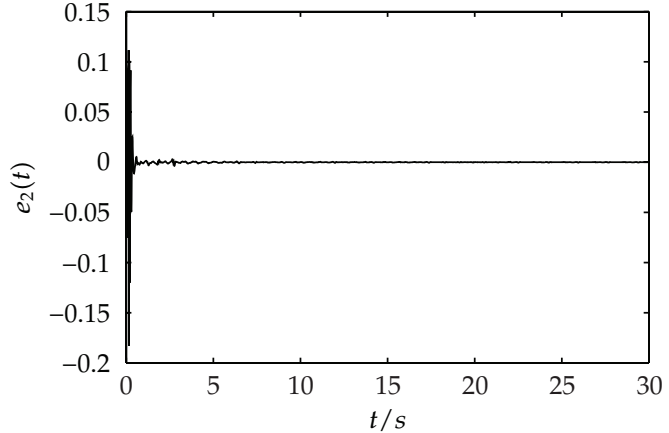

(b)

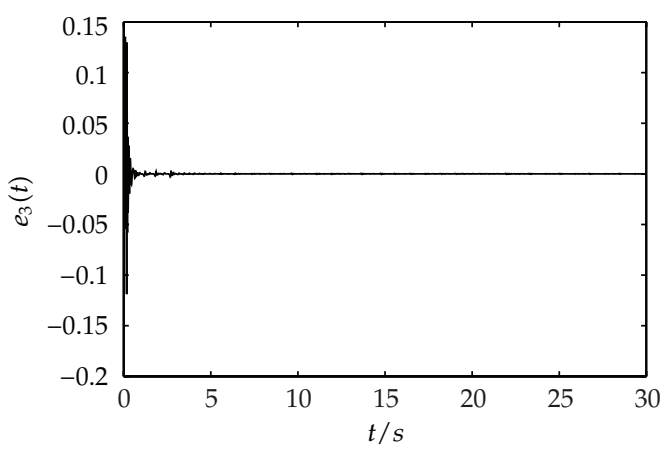

(c)

Figure 4: Error states of the drive system (3.1) and the response system (3.2).

and update laws of parameters are chosen as

$$
\begin{aligned}
& D_{*}^{q} \tilde{a}=\left(x_{2}-x_{1}\right) e_{1}-x_{1} e_{2}, \\
& D_{*}^{q} \tilde{b}=-x_{3} e_{3}, \\
& D_{*}^{q} \tilde{c}=\left(x_{1}+x_{2}\right) e_{2}, \\
& D_{*}^{q} \tilde{\alpha}=\left(y_{2}-y_{1}\right) e_{1}, \\
& D_{*}^{q} \tilde{\beta}=y_{2} e_{2}, \\
& D_{*}^{q} \tilde{\theta}=-y_{3} e_{3},
\end{aligned}
$$

then inverse synchronization between systems (3.1) and (3.2) will be obtained for any initial values, where $e_{a}=\tilde{a}-a, e_{b}=\tilde{b}-b, e_{c}=\tilde{c}-c, e_{\alpha}=\tilde{\alpha}-\alpha, e_{\beta}=\tilde{\beta}-\beta, e_{\theta}=\tilde{\theta}-\theta, \tilde{a}, \tilde{b}, \tilde{c}, \tilde{\alpha}, \tilde{\beta}$, and $\tilde{\theta}$ are the parameter estimations of $a, b, c, \alpha, \beta$, and $\theta$, respectively, and $e_{a}, e_{b}, e_{c}, e_{\alpha}, e_{\beta}$, and $e_{\theta}$ are the corresponding parameter errors.

\section{Numerical Simulations}

In this section, numerical simulations will be given to verify theoretical results obtained in the previous section. The true values of the "unknown" parameters of two uncertain systems 


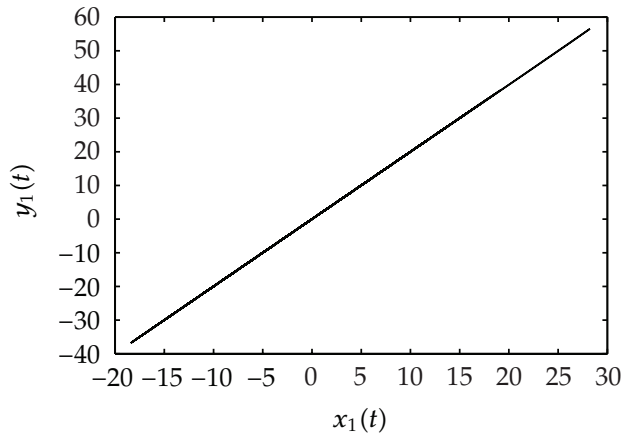

(a)

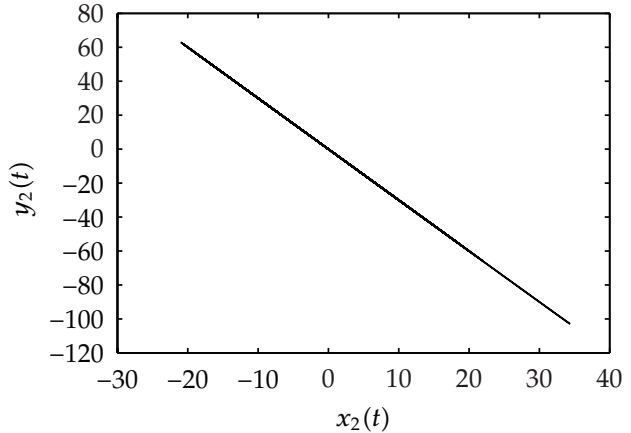

(b)

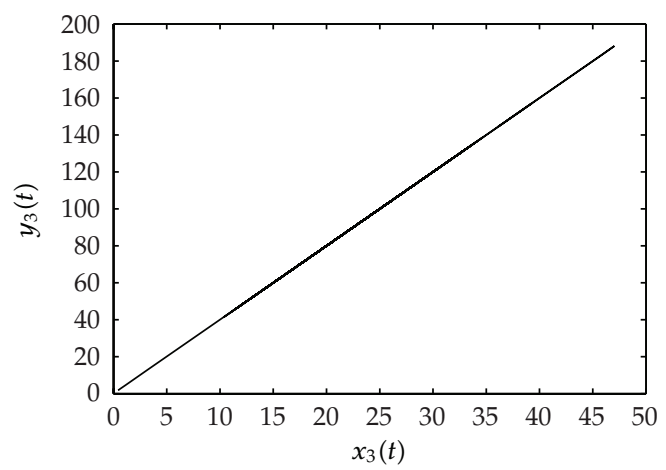

(c)

Figure 5: Signals $x_{1}(t)$ versus $y_{1}(t), x_{2}(t)$ versus $y_{2}(t)$, and $x_{3}(t)$ versus $y_{3}(t)$ after synchronization.

are taken as $a=35, b=3, c=28$ and $\alpha=36, \beta=20, \theta=3$. For this numerical simulation, the initial values of the drive and the response systems are chosen as $\left(x_{1}(0), x_{2}(0), x_{3}(0)\right)=$ $(0.3,0.4,0.5)$ and $\left(y_{1}(0), y_{2}(0), y_{3}(0)\right)=(0.4,0.5,0.6)$, respectively. Hence the initial values of the error system are $\left(e_{1}(0), e_{2}(0), e_{3}(0)\right)=(0.1,0.1,0.1)$, the initial estimated parameters $(\tilde{a}(0), \tilde{b}(0), \widetilde{c}(0), \tilde{\alpha}(0), \tilde{\beta}(0), \widetilde{\theta}(0))=(32,2,25,33,22,5)$. Without loss of generality, the scaling matrix $\sigma$ is chosen as $\operatorname{diag}(2,-3,4)$ and $q=0.9$. Figure 4 displays the synchronization error states between systems (3.1) and (3.2), respectively. Figure 5 shows the signals after synchronization. Figures 6 and 7 show that the parameter errors $e_{a}, e_{b}, e_{c}, e_{\alpha}, e_{\beta}, e_{\theta}$ converge to 0 , which means the estimated values of unknown parameters $\tilde{a}, \tilde{b}, \tilde{c}, \tilde{\alpha}, \tilde{\beta}, \tilde{\theta}$ converge to $a=25, b=3, c=28, \alpha=36, \beta=20$, and $\theta=3$ as $t \rightarrow \infty$, respectively. Figure 8 illustrates the projective chaotic attractors of drive system (3.1) and response system (3.2).

\section{Conclusion}

In this paper, adaptive projective synchronization of different chaotic systems with fully unknown parameters is presented. Based on the stability theory of fractional-order differential equations, a suitable adaptive controller and parameter update laws are designed to ensure fractional-order Chen system synchronize with fractional-order Lü system up to a scaling factor $\sigma$. Also the corresponding results are obtained to judge complete synchronization and inverse synchronization between fractional-order Chen system and 


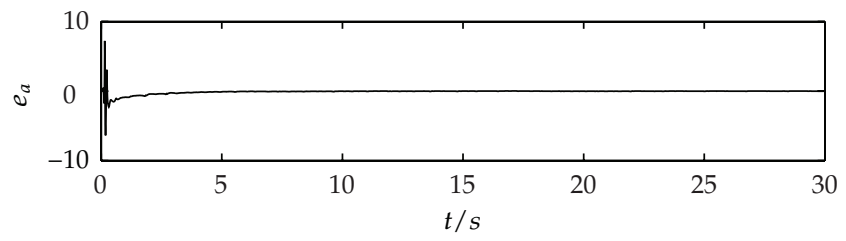

(a)

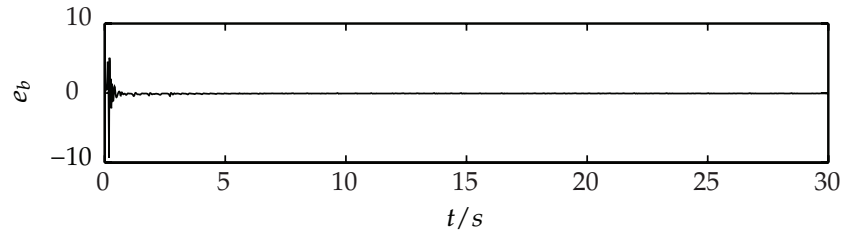

(b)

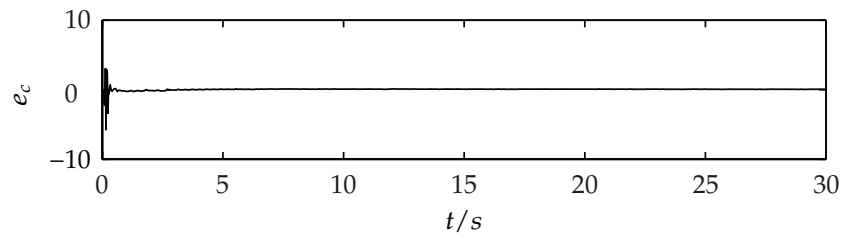

(c)

Figure 6: Parameter errors in the drive system (3.1), $e_{a}=\tilde{a}-a, e_{b}=\tilde{b}-b, e_{a}=\tilde{b}-b$.

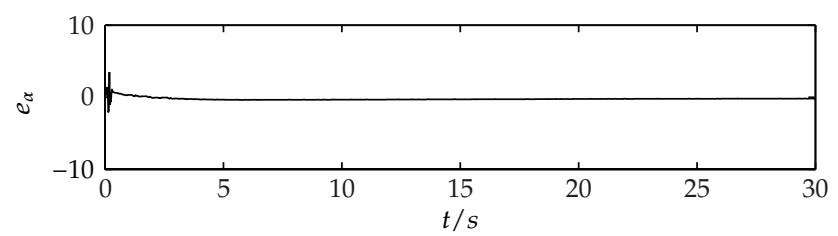

(a)

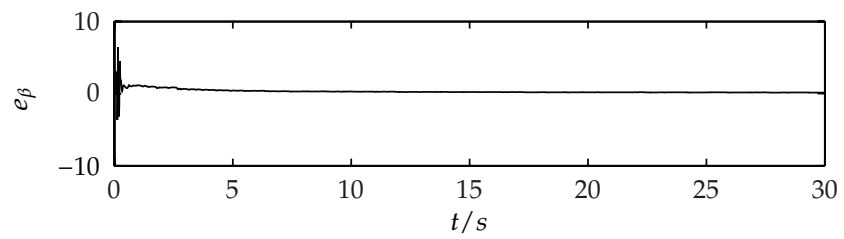

(b)

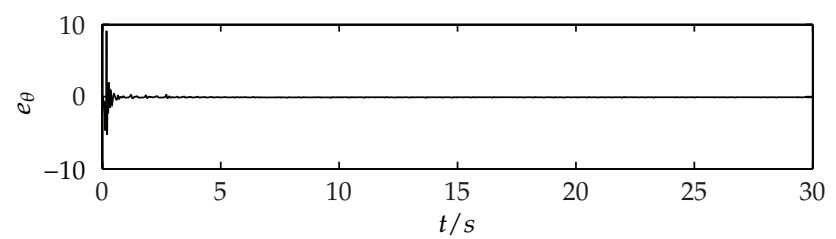

(c)

Figure 7: Parameter errors in the response system (3.2), $e_{\alpha}=\widetilde{\alpha}-\alpha, e_{\beta}=\widetilde{\beta}-\beta, e_{\theta}=\widetilde{\theta}-\theta$. 


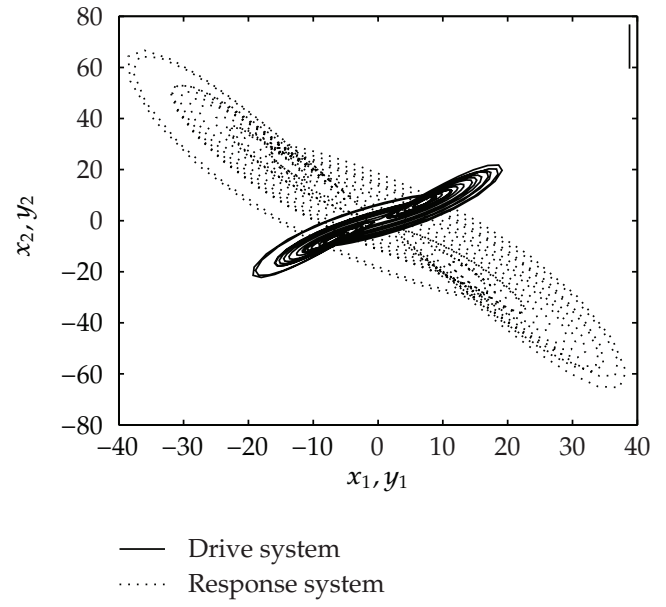

(a)

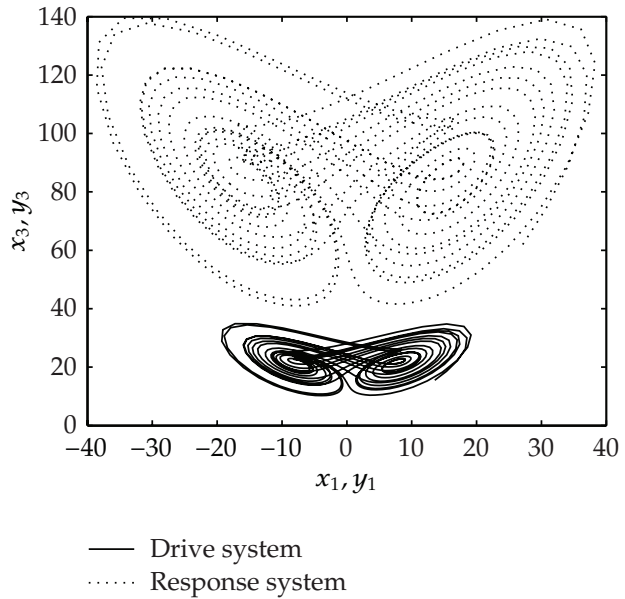

(b)

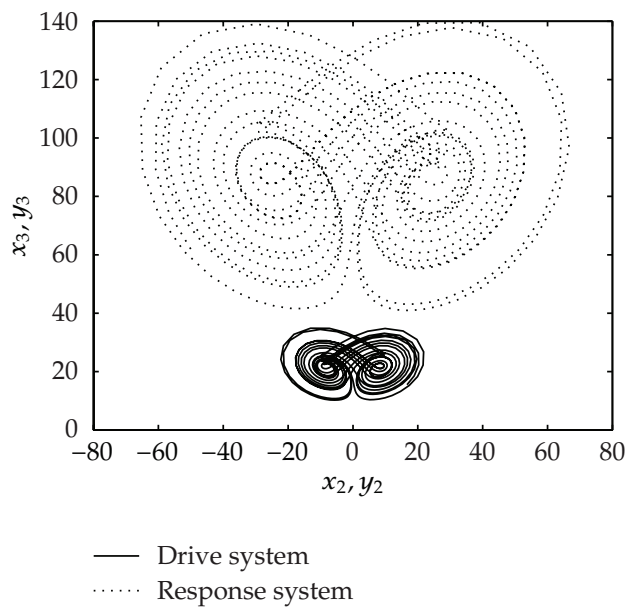

(c)

Figure 8: The projective attractors of drive system (3.1) and response system (3.2) $\left(\sigma_{1}=2, \sigma_{2}=-3, \sigma_{3}=4\right)$.

fractional-order Lü system, respectively. Meanwhile, results could be extended to other fractional-order chaotic or hyperchaotic systems, such as fractional-order Lorenz system and fractional-order Rössler system, fractional-order hyperchaotic Chen system, and fractionalorder hyperchaotic Lü system.

\section{Acknowledgments}

This work is supported by the National Natural Science Foundation of China (no. 60974090), the Fundamental Research Funds for the Central Universities (no. CDJXS11172237), the Specialized Research Fund for the Doctoral Program of Higher Education of China (nos. 20093401120001; 102063720090013), the Natural Science Foundation of Anhui Province (no. 11040606M12), the Natural Science Foundation of Anhui Education Bureau (no. KJ2010A035), PhD. Candidate Academic Foundation of Ministry of Education of China and the 211 project of Anhui University. 


\section{References}

[1] G. Chen and X. Dong, From Chaos to Order-Perspectives, Methodologies and Applications, World Scientific, Singapore, 1998.

[2] T. Yang and L. O. Chua, "Impulsive stabilization for control and synchronization of chaotic systems: theory and application to secure communication," IEEE Transactions on Circuits and Systems I, vol. 44, no. 10, pp. 976-988, 1997.

[3] S. K. Dana, P. K. Roy, and J. Kurths, Complex Dynamics in Physiological Systems: From Heart to Brain, Springer, NewYork, NY, USA, 2009.

[4] J. García-Ojalvo and R. Roy, "Spatiotemporal communication with synchronized optical chaos," Physical Review Letters, vol. 86, no. 22, pp. 5204-5207, 2001.

[5] J. C. Sprott, Chaos and Time-Series Analysis, Oxford University Press, Oxford, UK, 2003.

[6] G. M. Mahmoud and E. E. Mahmoud, "Complete synchronization of chaotic complex nonlinear systems with uncertain parameters," Nonlinear Dynamics, vol. 62, no. 4, pp. 875-882, 2010.

[7] L. Wang, Z. Yuan, X. Chen, and Z. Zhou, "Lag synchronization of chaotic systems with parameter mismatches," Communications in Nonlinear Science and Numerical Simulation, vol. 16, no. 2, pp. 987992, 2011.

[8] G. H. Erjaee and S. Momani, "Phase synchronization in fractional differential chaotic systems," Physics Letters A, vol. 372, no. 14, pp. 2350-2354, 2008.

[9] X. R. Shi and Z. L. Wang, "Adaptive added-order anti-synchronization of chaotic systems with fully unknown parameters," Applied Mathematics and Computation, vol. 215, no. 5, pp. 1711-1717, 2009.

[10] J. W. Wang and A. M. Chen, "Partial synchronization in coupled chemical chaotic oscillators," Journal of Computational and Applied Mathematics, vol. 233, no. 8, pp. 1897-1904, 2010.

[11] S. Y. Li and Z. M. Ge, "Generalized synchronization of chaotic systems with different orders by fuzzy logic constant controller," Expert Systems with Applications, vol. 38, no. 3, pp. 2302-2310, 2011.

[12] X. Liu, "Impulsive synchronization of chaotic systems subject to time delay," Nonlinear Analysis, Theory, Methods and Applications, vol. 71, no. 12, pp. e1320-e1327, 2009.

[13] J. M. González-Miranda, "Synchronization of symmetric chaotic systems," Physical Review E, vol. 53, no. 6, pp. 5656-5669, 1996.

[14] R. Mainieri and J. Rehacek, "Projective synchronization in three-dimensional chaotic systems," Physical Review Letters, vol. 82, no. 15, pp. 3042-3045, 1999.

[15] D. Ghosh, "Generalized projective synchronization in time-delayed systems: nonlinear observer approach," Chaos, vol. 19, no. 1, Article ID 013102, 2009.

[16] G. H. Li, "Modified projective synchronization of chaotic system," Chaos, Solitons and Fractals, vol. 32, no. 5, pp. 1786-1790, 2007.

[17] Y. Chen and X. Li, "Function projective synchronization between two identical chaotic systems," International Journal of Modern Physics C, vol. 18, no. 5, pp. 883-888, 2007.

[18] H. Du, Q. Zeng, and C. Wang, "Modified function projective synchronization of chaotic system," Chaos, Solitons and Fractals, vol. 42, no. 4, pp. 2399-2404, 2009.

[19] C. Y. Chee and D. Xu, "Chaos-based M-ary digital communication technique using controlled projective synchronisation," IEE Proceedings: Circuits, Devices and Systems, vol. 153, no. 4, pp. 357-360, 2006.

[20] Y. Yu and H. X. Li, "Adaptive hybrid projective synchronization of uncertain chaotic systems based on backstepping design," Nonlinear Analysis, vol. 12, no. 1, pp. 388-393, 2011.

[21] R. Z. Luo, S. C. Deng, and Z. M. Wei, "Modified projective synchronization between two different hyperchaotic systems with unknown or/and uncertain parameters," Physica Scripta, vol. 81, no. 1, Article ID 015006, 2010.

[22] H. Du, Q. Zeng, and N. Lü, “A general method for modified function projective lag synchronization in chaotic systems," Physics Letters A, vol. 374, no. 13-14, pp. 1493-1496, 2010.

[23] K. S. Sudheer and M. Sabir, "Function projective synchronization in chaotic and hyperchaotic systems through open-plus-closed-loop coupling," Chaos, vol. 20, no. 1, Article ID 013115, pp. 1-5, 2010.

[24] R. C. Koeller, "Polynomial operators, Stieltjes convolution, and fractional calculus in hereditary mechanics," Acta Mechanica, vol. 58, no. 3-4, pp. 251-264, 1986.

[25] R. C. Koeller, "Applications of fractional calculus to the theory of viscoelasticity," Journal of Applied Mechanics, vol. 51, no. 2, pp. 299-307, 1984.

[26] O. Heaviside, Electromagnetic Theory, Chelsea, New York, NY, USA, 1971. 
[27] T. T. Hartley, C. F. Lorenzo, and H. K. Qammer, “Chaos in a fractional order Chua's system," IEEE Transactions on Circuits and Systems I, vol. 42, no. 8, pp. 485-490, 1995.

[28] C. Li and G. Chen, "Chaos and hyperchaos in the fractional-order Rössler equations," Physica A, vol. 341, no. 1-4, pp. 55-61, 2004.

[29] C. Li and G. Peng, "Chaos in Chen's system with a fractional order," Chaos, Solitons and Fractals, vol. 22, no. 2, pp. 443-450, 2004.

[30] W. H. Deng and C. P. Li, "Chaos synchronization of the fractional Lü system," Physica A, vol. 353, no. 1-4, pp. 61-72, 2005.

[31] Z. M. Ge and C. Y. Ou, "Chaos in a fractional order modified Duffing system," Chaos, Solitons and Fractals, vol. 34, no. 2, pp. 262-291, 2007.

[32] X. Wang and Y. He, "Chaotic synchronization of fractional-order modified coupled dynamos system," International Journal of Modern Physics B, vol. 23, no. 31, pp. 5769-5777, 2009.

[33] M. M. Asheghan, M. T. H. Beheshti, and M. S. Tavazoei, “Robust synchronization of perturbed Chen's fractional order chaotic systems," Communications in Nonlinear Science and Numerical Simulation, vol. 16, no. 2, pp. 1044-1051, 2011.

[34] D. Cafagna and G. Grassi, “Observer-based synchronization for a class of fractional chaotic systems via a scalar signal: results involving the exact solution of the error dynamics," International Journal of Bifurcation and Chaos in Applied Sciences and Engineering, vol. 21, no. 3, pp. 955-962, 2011.

[35] S. Wang, Y. Yu, and M. Diao, "Hybrid projective synchronization of chaotic fractional order systems with different dimensions," Physica A, vol. 389, no. 21, pp. 4981-4988, 2010.

[36] X. Wu and H. Wang, "A new chaotic system with fractional order and its projective synchronization," Nonlinear Dynamics, vol. 61, no. 3, pp. 407-417, 2010.

[37] G. Peng, Y. Jiang, and F. Chen, "Generalized projective synchronization of fractional order chaotic systems," Physica A, vol. 387, no. 14, pp. 3738-3746, 2008.

[38] D. Cafagna and G. Grassi, "Observer-based projective synchronization of fractional systems via a scalar signal: application to hyperchaotic RÄossler systems," Nonlinear Dynamics. In press.

[39] L. D. Zhao, J. B. Hu, and X. H. Liu, "Adaptive tracking control and synchronization of fractional hyper-chaotic Lorenz system with unknown parameters," Acta Physica Sinica, vol. 59, no. 4, pp. 23052309, 2010.

[40] D. Cafagna, "Past and present-Fractional calculus: a mathematical tool from the past for present engineers," IEEE Industrial Electronics Magazine, vol. 1, no. 2, pp. 35-40, 2007.

[41] R. Gorenflo and F. Mainardi, Fractional Calculus: Integral and Differential Equations of Fractional Order: Fractal and Fractional Calculus in Continuum Mechanics, Springer, Wien, Germany, 1997.

[42] K. Diethelm, N. J. Ford, and A. D. Freed, "A predictor-corrector approach for the numerical solution of fractional differential equations," Nonlinear Dynamics, vol. 29, no. 1-4, pp. 3-22, 2002.

[43] K. Diethelm, N. J. Ford, and A. D. Freed, "Detailed error analysis for a fractional Adams method," Numerical Algorithms, vol. 36, no. 1, pp. 31-52, 2004.

[44] K. Diethelm and N. J. Ford, "Analysis of fractional differential equations," Journal of Mathematical Analysis and Applications, vol. 265, no. 2, pp. 229-248, 2002.

[45] C. Li and G. Chen, "Chaos in the fractional order Chen system and its control," Chaos, Solitons and Fractals, vol. 22, no. 3, pp. 549-554, 2004.

[46] J. Wang, X. Xiong, and Y. Zhang, "Extending synchronization scheme to chaotic fractional-order Chen systems," Physica A, vol. 370, no. 2, pp. 279-285, 2006.

[47] H. Zhu, S. Zhou, and Z. He, "Chaos synchronization of the fractional-order Chen's system," Chaos, Solitons and Fractals, vol. 41, no. 5, pp. 2733-2740, 2009.

[48] J. G. Lu, "Chaotic dynamics of the fractional-order Lü system and its synchronization," Physics Letters, Section A, vol. 354, no. 4, pp. 305-311, 2006.

[49] D. Matignon, "Stability results of fractional differential equations with applications to control processing," in Proceedings of the IEEE-SMC International Association for Mathematics and Computers in Simulation (IMACS '96), pp. 963-968, Lille, France, 1996. 


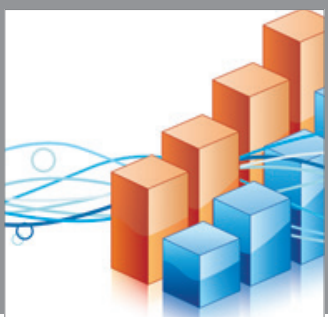

Advances in

Operations Research

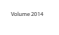

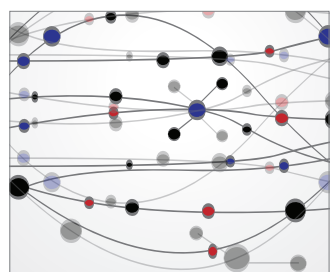

\section{The Scientific} World Journal
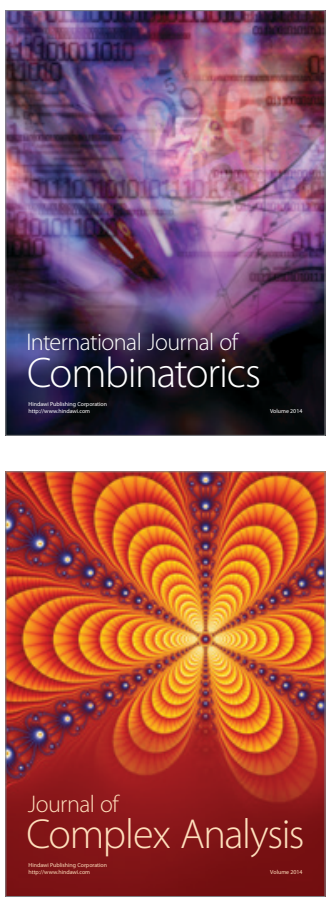

International Journal of

Mathematics and

Mathematical

Sciences
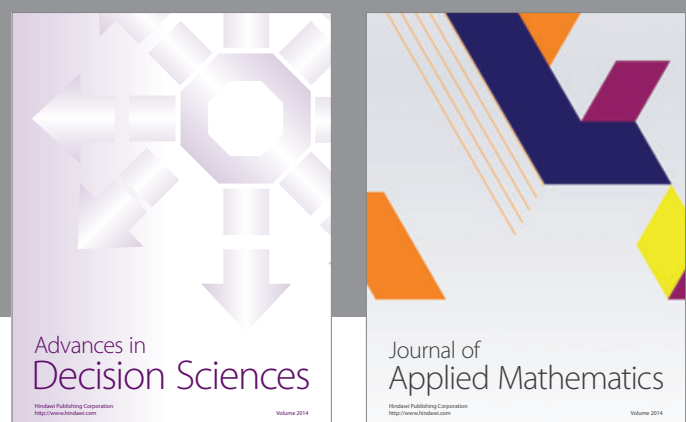

Journal of

Applied Mathematics
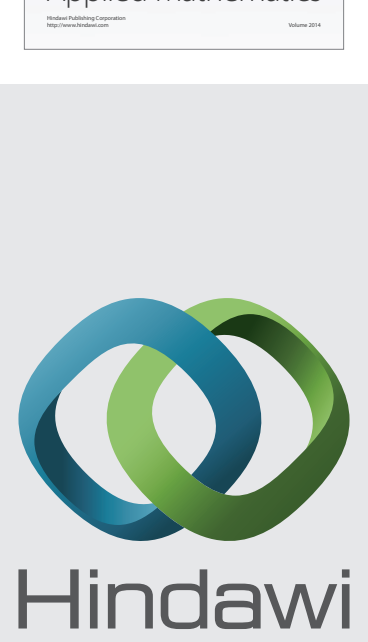

Submit your manuscripts at http://www.hindawi.com
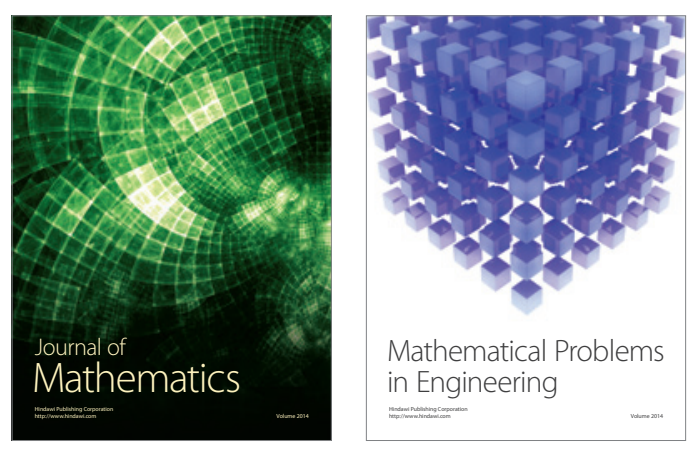

Mathematical Problems in Engineering
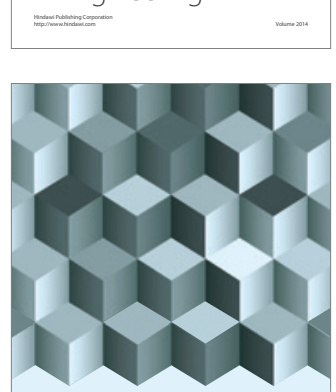

Journal of

Function Spaces
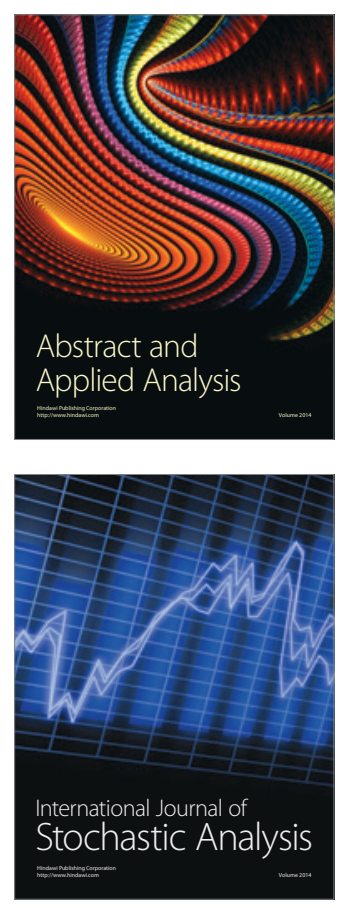

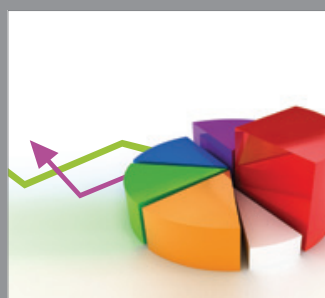

ournal of

Probability and Statistics

Promensencen
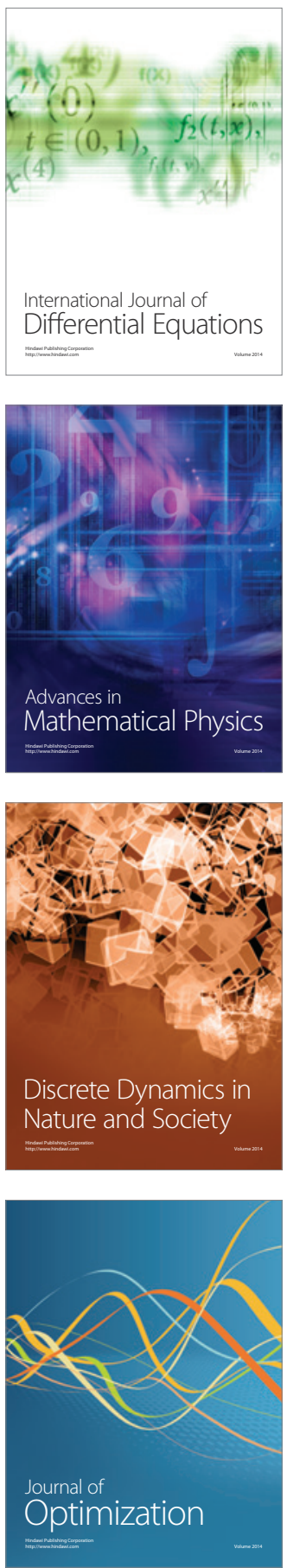